\title{
Does denosumab offer survival benefits? -Our experience with denosumab in metastatic non-small cell lung cancer patients treated with immune-checkpoint inhibitors
}

\author{
Yenong $\mathrm{Cao}^{1}$, Muhammad Zubair Afzal ${ }^{2}$, Keisuke Shirai ${ }^{2}$ \\ ${ }^{1}$ Department of Internal Medicine, Dartmouth-Hitchcock Medical Center, One Medical Center Dr., Lebanon, NH, USA; ${ }^{2}$ Department of \\ Hematology-Oncology, Norris Cotton Cancer Center, One Medical Center Dr., Lebanon, NH, USA \\ Contributions: (I) Conception and design: All authors; (II) Administrative support: None; (III) Provision of study materials or patients: K Shirai; (IV) \\ Collection and assembly of data: Y Cao, MZ Afzal; (V) Data analysis and interpretation: All authors; (VI) Manuscript writing: All authors; (VII) Final \\ approval of manuscript: All authors. \\ Correspondence to: Yenong Cao, MD, PhD. Department of Internal Medicine, Dartmouth-Hitchcock Medical Center, One Medical Center Dr., \\ Lebanon, NH 03756, USA. Email: Yenong.Cao@hitchcock.org.
}

Background: Immune checkpoint inhibitors (ICIs) have revolutionized the treatment of non-small-cell lung cancer (NSCLC). Denosumab is a humanized monoclonal antibody to RANK ligand used to prevent skeletal-related events of bone metastases in solid tumors. We are reporting the clinical outcomes in our NSCLC patients who received RANKL inhibitor in combination with ICIs.

Methods: This observational study used retrospective data from a tertiary cancer center from 2015-2020. Stage IV non-small cell lung cancer patients who received denosumab within 30 days of ICIs (pembrolizumab, nivolumab, atezolizumab, ipilimumab) were included. Kaplan-Meier curves were obtained for survival analysis.

Results: We identified 69 patients and all had skeletal metastases, and 37.7\% had brain metastases. Median OS was 6.3 months and median PFS was 2.8 months, with overall response rate (ORR) of $18.8 \%$ and disease control rate (DCR) of $40.6 \%$. Median OS in patients with concomitant denosumab and ICIs more than 3 months was 11.5 months, comparing to 3.6 months in patients with $<3$ months of concomitant therapy $(\mathrm{P}=0.0005)$. OS and PFS did not differ with respect to brain metastases or number of skeletal metastases. However, the duration of ICIs and denosumab overlap was associated with improved OS and PFS. Among the $18.8 \%$ of patients who achieved complete response (CR) and partial response (PR), six-month survival rate was $100 \%$ and one-year survival rate was $69.2 \%$. Most of the patients tolerated denosumab well, and hypocalcemia was the most commonly reported side effect.

Conclusions: Patients receiving combination therapy did not perform poorly comparing to published studies despite of poor prognostic features such as brain metastases and numerous skeletal metastases. Although we did notice potential benefit of the longer duration of concomitant use of ICI and denosumab, future prospective clinical trials are needed to evaluate the synergistic effect of RANKL inhibitors/ICI and if duration of RANKL inhibitors matters.

Keywords: Non-small cell lung cancer (NSCLC); immune checkpoint inhibitors (ICIs); RANKL inhibitors; denosumab

Submitted Jan 28, 2021. Accepted for publication May 14, 2021.

doi: $10.21037 /$ jtd-21-150

View this article at: https://dx.doi.org/10.21037/jtd-21-150 


\section{Introduction}

Lung cancer is the leading cause of cancer-related mortality in USA. For all stages combined, the 5-year survival rate is $19.0 \%$. Low lung cancer survival rates is explained by the large portion of patients (57\%) diagnosed with metastatic disease, for which the 5 -year survival rate is $5 \%$ (1). National Cancer Institute reports that $80 \%$ of lung cancers are non-small cell lung cancer (NSCLC) (2). In NSCLC, monoclonal antibodies targeting PD-1/PDL1, such as pembrolizumab, nivolumab and atezolizumab, along with monoclonal antibodies targeting CTLA-4, such as ipilimumab, have shown promising results. Nivolumab has demonstrated improvement of progression-free survival (PFS) and response rate compared to docetaxel in NSCLC (3). FDA approved pembrolizumab and nivolumab in 2015 to be used in NSCLC. In KEYNOTE-189, median progression-free survival was 8.8 months for pembrolizumab plus carboplatin/pemetrexed and 4.9 months for chemotherapy only. $47.6 \%$ patients in the pembrolizumab plus chemotherapy group achieved an objective response compared with $18.9 \%$ patients in the chemotherapy only group (4).

Receptor activator of nuclear factor (NF)- $\kappa \mathrm{B}$ ligand (RANKL) and its associated receptor RANK are important for the osteoclastogenesis of the bone (5). Since RANKL is known to be essential for osteoclastogenesis, investigators have targeted this ligand for the prevention of the metastatic bone disease. Denosumab is a humanized $\mathrm{mAb}$ to RANKL. It inhibits the RANK-RANKL interaction and results in decreased osteoclast-induced bone resorption. Denosumab is approved by FDA in 2010 for the treatment of osteoporosis and for prevention of skeletal events from solid tumors (6). It has also been shown to be non-inferior to zoledronic acid (bisphosphonate) in treatment of skeletal related complications (7). Lung cancer has a great potential to metastasize. Approximately $40 \%$ of the patients with lung cancers develop bone metastasis and $22 \%$ to $59 \%$ patients with bone metastasis experience skeletal related events (8). Denosumab (Anti-RANKL) is thought to have direct or indirect antitumor effects. There is growing evidence suggesting a role of RANK/RANKL signaling in tumorigenesis. These 2 proteins are overexpressed in tumor microenvironments. Blockage of these proteins in animal models have resulted in decreasing incidence of skeletal related events and improving survival in solid tumors including lung cancer (9). In a post-hoc analysis of phase III clinical trial in metastatic lung cancer patients, denosumab was associated with improved overall survival comparing to zoledronic acid (10).

Though Anti-RANKL is being used in NSCLC in combination with immune checkpoint inhibitors (ICI), there have been scant reported studies to evaluate the synergistic antitumor effect of ICI and anti-RANKL in the literature. In this study, we aim to evaluate the patient's response to combination therapy with Anti-RANKL and ICI in NSCLC.

We present the following article in accordance with the STROBE reporting checklist (available at https://dx.doi. org/10.21037/jtd-21-150).

\section{Methods}

This study was conducted at Dartmouth Hitchcock Medical Center (DHMC) in conjunction with Norris Cotton Cancer Center. Electronic medical records were accessed. Patients who carry the diagnosis of NSCLC who have received ICI (ipilimumab, pembrolizumab, nivolumab, atezolizumab) either alone or in combination with chemotherapy as well as concurrent (defined as either simultaneously or within one month of starting or stopping ICI) denosumab were identified. We used the timeframe from January $1^{\text {st }}, 2015$ until October $30^{\text {th }} 2020$. The only exclusion criterion was the patients $<18$ years of age. The study was conducted in accordance with the Declaration of Helsinki (as revised in 2013). The study was approved by institutional review board of Dartmouth-Hitchcock (NO.: STUDY00030057) and individual consent for this retrospective analysis was waived.

This was a retrospective study. We obtained data including basic demographics (age, sex, race), time of diagnosis, histological sub-type (adenocarcinoma, squamous cell carcinoma), AJCC clinical stage at ICI initiation, type of chemotherapy and ICI, duration of ICI therapy, prior chemotherapy, radiation therapy, duration of denosumab, duration of concurrent denosumab and ICI, date of death, date of progression, mutations, tumor proportion score (TPS) for PD-L1, metastatic status, number of metastatic sites involved, brain metastases. Best radiographic response, i.e., complete remission (CR), partial response (PR), stable disease (SD) and progressive disease (PD) were recorded per RECIST v1.1 criteria. Progression of disease, current status (dead/alive) were recorded as well. Overall response rate (ORR) was calculated and defined as the percentage of patients who achieved either PR or CR. Disease control rate (DCR) was defined as the total percentage of patients achieving CR, PR, and SD. OS [from the initiation of the 
therapy till the date of last follow-up (October $\left.30^{\text {th }}, 2020\right)$, or the date patient passed away], PFS [from the date of therapy initiation till the last follow up (October $\left.30^{\text {th }}, 2020\right)$, date of progression or date of death)] were calculated. We also recorded the $\mathrm{LDH}$, performance status and side effects from denosumab. Side effects measures included skin rash/ mucositis, hypocalcemia, diarrhea/colitis, hepatotoxicity, pneumonitis, arthralgia, neuro and cardiotoxicity. We grouped patients by brain metastases and no brain metastases, skeletal metastatic sites of $>3$, TPS of $\leq 1 \%$, $1-49 \%, \geq 50 \%)$. No patient lost to follow up and there were no missing data points.

\section{Statistical analysis}

Summary measures of continuous data such as age at diagnosis of advanced stage disease, OS, PFS, LDH, mean (or geometric mean, as appropriate), median, standard deviation (SD) and inter-quartile range were calculated. Histograms and qq-plots of continuous endpoints were used to evaluate distributional assumptions. To evaluate the survival analysis (OS and PFS, with 95\% CI), the Kaplan-Meier method and the log-rank test were applied. The cox proportional hazards regression model was applied to perform univariable and multivariable analyses. Variables included in multivariate analysis were age at ICI initiation, sex, number of skeletal metastases, brain metastases, total number of metastatic sites involved, prior chemotherapy, radiation therapy, type of ICIs and TPS. Chi-square and Fisher exact tests were applied to compare the categorical variables and calculate the $\mathrm{P}$-value. Student t-tests were applied to analyze the continuous variables and to calculate the $\mathrm{P}$-values. Statistical significance is defined as $\mathrm{P}<0.05$. Logistic regression was performed to calculate Odds ratio. No sensitivity analysis was performed. All statistical analyses were performed using statistical software STATA Pro V. 14.2.

Independent variables were age, sex, TPS, anatomical sites involved with metastases, prior chemotherapy, type of ICIs. Dependent variables are the best response (BR), ORR, OS, PFS.

\section{Results}

There were total of 69 patients with NSCLC who received ICI and denosumab concurrently. $84 \%$ of the patients had adenocarcinoma. Slightly higher proportion of the patients were male $(53.6 \%$ vs. $46.4 \%)$ and majority of the patients were under 75 years of age ( $85.5 \%$ vs. $14.5 \%)$. Majority
$(89.8 \%)$ of the patients received ICI in combination with chemotherapy either first or second line. Only 7 (10.2\%) patients received ICI (pembrolizumab) as monotherapy because of high PD-L1 score or beyond first line of therapy. Median duration of ICI therapy was 2.5 months (1-34.5 months). Almost equal proportion of the patients received ICI as first or $2^{\text {nd }}$ line of therapy $(46.4 \% v s$. $43.5 \%)$. All patients received ICI as second line therapy after progressing on first line chemotherapy. Majority of the patients received pembrolizumab (85.5\%). PD-L1 TPS score is also given in Table 1. Patients with PD-L1 $<1 \%$ comprised $31.9 \%$ of the cohort population and $44.9 \%$ of patients had PD-L1 score $1-49 \%$. 23.2\% of patients had PD-L1 score of $\geq 50 \%$. Median duration of denosumab therapy was 5 months (1-34 months). Median duration of denosumab and ICI overlap therapy was 1.5 months (1-34 months). Overall, 11 (15.9\%) of the patients received prior chemotherapy and $47(68.1 \%)$ of the patients received radiation therapy. Median number of metastatic sites was 4 [1-15]. All patients have skeletal metastases and majority of the patients had $<3$ skeletal metastases ( $62.3 \%$ vs. $37.7 \%)$. Considerable number of patients had brain metastases as well (37.7\%). $81.3 \%$ patients had elevated LDH. Most of the patients tolerated denosumab well, however hypocalcemia was the most commonly reported side effect $(37.7 \%)$. One patient developed osteonecrosis of the jaw (Table 1).

\section{Survival analysis}

Overall, 57 (82.6\%) patients passed away by the time of last follow up. Median OS was 6.3 months. On univariate and multivariate analyses, TPS score, duration of denosumab and ICI overlap, and line of therapy had significant effect on the OS (Table 1). There was no difference in OS based on the age, sex, type of immunotherapy, radiation therapy, number of metastatic sites and elevated LDH. Median OS in patients receiving denosumab + ICI $>3$ months was 11.5 vs. 3.6 months in patients with shorter concurrent therapy $[\mathrm{P}=0.0005$ (2-5.3); Figure $1 A]$. Among the 13 patients who achieved CR and PR, six-month survival rate was $100 \%$ and one-year survival rate was $69.2 \%$. Patients with no brain metastases had better median OS but the difference was not statistically significant (7.7 vs. 3.6 months, $\mathrm{P}=0.16$; Figure $1 B)$. Median OS was significantly higher in patients who received ICI as first line therapy (8.9 vs. 3.9 months, $\mathrm{P}=0.03$; Figure 1C). Similarly, the patient with $>3$ skeletal metastases had lower OS, but the difference was not statistically significant as well (7.7 vs. 
Table 1 Overall survival (univariate and multivariate analysis) and patient characteristics

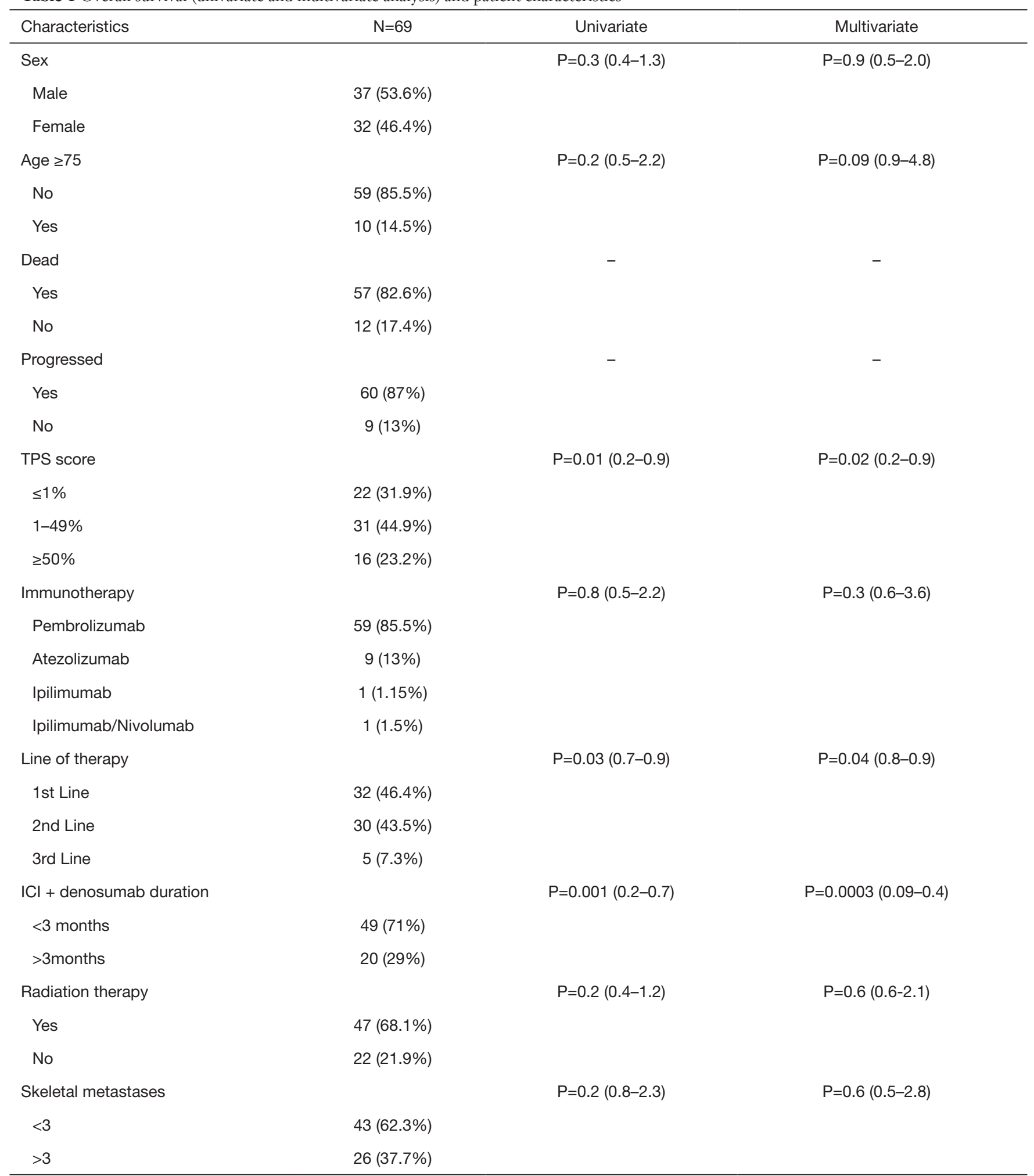

Table 1 (continued) 
Table 1 (continued)

\begin{tabular}{|c|c|c|c|}
\hline Characteristics & $\mathrm{N}=69$ & Univariate & Multivariate \\
\hline Yes & $43(62.3 \%)$ & & \\
\hline No & $26(37.7 \%)$ & & \\
\hline Number of metastatic sites & & $P=0.3(0.8-2.3)$ & $P=0.2(0.6-5.6)$ \\
\hline$>3$ sites & $41(59.4 \%)$ & & \\
\hline Hypocalcemia & & - & - \\
\hline Yes & $26(37.7 \%)$ & & \\
\hline No & $63(62.3 \%)$ & & \\
\hline$<220$ IU & $12(18.7 \%)$ & & \\
\hline
\end{tabular}

TPS, tumor proportion score; ICI, immune checkpoint inhibitors; LDH, lactate dehydrogenase.
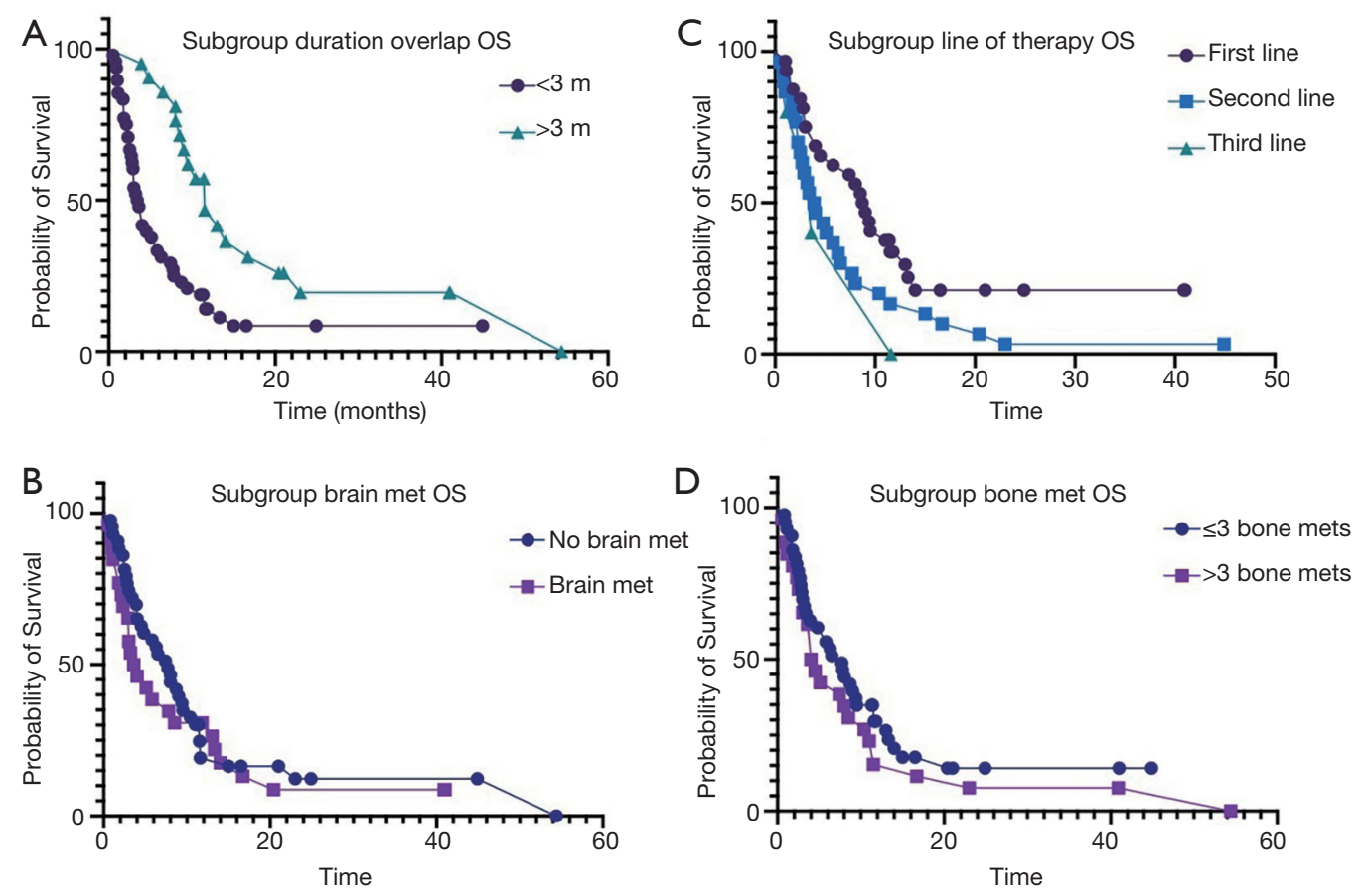

Figure 1 Kaplan-Meier estimate of OS. (A) Kaplan-Meier estimate of OS subgroups by duration of denosumab and ICI overlap (11.5 vs. 3.6 months, $\mathrm{P}=0.0005)$. (B) Kaplan-Meier estimate of OS subgroups by brain metastases (7.7 vs. 3.6 months, $\mathrm{P}=0.16)$. (C) Kaplan-Meier estimate of OS subgroups by line of therapy i.e., first line vs. second line (8.9 vs. 3.9 months, $\mathrm{P}=0.03$ ). (D) Kaplan-Meier estimate of OS subgroups by skeletal metastases ( 7.7 vs. 4 months, $\mathrm{P}=0.2)$. ICI, immune checkpoint inhibitors. 
Table 2 Progression free survival (univariate and multivariate analysis)

\begin{tabular}{|c|c|c|}
\hline Characteristics & Univariate & Multivariate \\
\hline Sex & \multirow[t]{3}{*}{$P=0.3(0.4-1.3)$} & \multirow[t]{3}{*}{$P=0.6(0.5-2.4)$} \\
\hline Male & & \\
\hline Female & & \\
\hline Age $\geq 75$ & \multirow[t]{3}{*}{$P=0.5(0.3-1.6)$} & \multirow[t]{3}{*}{$\mathrm{P}=0.4(0.6-3.3)$} \\
\hline Yes & & \\
\hline No & & \\
\hline TPS score & \multirow[t]{5}{*}{$P=0.06(0.08-0.65)$} & \multirow[t]{5}{*}{$P=0.06(0.2-1.0)$} \\
\hline $0 \%$ & & \\
\hline$<1 \%$ & & \\
\hline $1-49 \%$ & & \\
\hline$\geq 50 \%$ & & \\
\hline Immunotherapy & \multirow[t]{5}{*}{$P=0.5(0.4-1.6)$} & \multirow[t]{5}{*}{$P=0.8(0.4-2.4)$} \\
\hline Pembrolizumab & & \\
\hline Atezolizumab & & \\
\hline Ipilimumab & & \\
\hline Ipilimumab/nivolumab & & \\
\hline Line of therapy & $P=0.006(0.2-0.5)$ & $\mathrm{P}=0.005(0.7-0.9)$ \\
\hline $\begin{array}{l}\mathrm{ICl}+\text { denosumab } \\
\text { duration }\end{array}$ & \multirow[t]{3}{*}{$P=0.006(0.2-0.7)$} & \multirow[t]{3}{*}{$P=0.0002(0.1-0.4)$} \\
\hline$<3$ months & & \\
\hline$>3$ months & & \\
\hline Radiation therapy & \multirow[t]{3}{*}{$P=0.2(0.4-1.2)$} & \multirow[t]{3}{*}{$P=0.7(0.5-2.0)$} \\
\hline Yes & & \\
\hline No & & \\
\hline Skeletal metastases & \multirow[t]{3}{*}{$P=0.1(0.8-2.5)$} & \multirow[t]{3}{*}{$\mathrm{P}=0.7(0.4-2.7)$} \\
\hline$<3$ & & \\
\hline$>3$ & & \\
\hline Brain metastases & \multirow[t]{3}{*}{$P=0.3(0.7-2.2)$} & \multirow[t]{3}{*}{$P=0.3(0.6-3.24)$} \\
\hline Yes & & \\
\hline No & & \\
\hline $\begin{array}{l}\text { Number of metastatic } \\
\text { sites }\end{array}$ & \multirow[t]{3}{*}{$P=0.05(0.9-2.9)$} & \multirow[t]{3}{*}{$P=0.03(1.1-10.1)$} \\
\hline $1-3$ sites & & \\
\hline$>3$ sites & & \\
\hline
\end{tabular}

Table 2 (continued)
Table 2 (continued)

\begin{tabular}{lll}
\hline Characteristics & Univariate & Multivariate \\
\hline LDH & $\mathrm{P}=0.5(0.6-2.4)$ & $\mathrm{P}=0.3(0.2-1.5)$ \\
$>220 \mathrm{IU}$ & & \\
$<220 \mathrm{IU}$ & & \\
\hline
\end{tabular}

TPS, tumor proportion score; ICl, immune checkpoint inhibitors; $\mathrm{LDH}$, lactate dehydrogenase.

4 months, $\mathrm{P}=0.2$; Figure $1 D$ ). Median OS in PD-L1 $\geq 1 \%$ group was 7.4 vs. 4.4 months in $\mathrm{PD}-\mathrm{L} 1<1 \%$ group.

Overall, $60(87 \%)$ patients progressed while on therapy. Median overall PFS was 2.8 months. On univariate and multivariate analyses, duration of denosumab + ICI overlap (0.0002, 95\% CI: $0.1-0.4)$, line of therapy $(0.005,95 \%$ CI: $0.7-0.9)$ and number of metastatic sites $(0.03,95 \%$ CI: 1.1-10.1) had significant impact on the PFS (Table 2). Median PFS in patients receiving denosumab + ICI $>3$ months was 6.0 vs. 1.9 months $(\mathrm{P}=0.0005$; Figure $2 A)$. There was no significant difference in median PFS in patients with brain metastases (3.9 vs. 2.1 months, $\mathrm{P}=0.2$; Figure $2 B$ ). The patients who received ICI as first line therapy had significantly higher median PFS (4.3 vs. 1.8 months, $\mathrm{P}=0.006$; Figure $2 C$ ). There was also no difference in median PFS in patient who had $>3$ skeletal metastases (3.7 vs. 2 months, $\mathrm{P}=0.1$; Figure 2D).

\section{Radiographic response}

Majority of the patients had progressive disease (59.4\%). Three $(4.3 \%)$ patients had complete response (CR), 10 (14.5\%) patients had partial response (PR) and 15 (21.8\%) patients had stable disease (SD). The overall response rate (ORR) was $18.8 \%$ whereas the disease control rate (DCR) was $40.6 \%$ (Table 3). Duration of concurrent denosumab and ICI had significant effect on radiographic response. The odds ratio for ORR in patients with denosumab+ ICI $>3$ months was $3.85, \mathrm{P}=0.03(1.1-13.5)$ and the odds ratio for DCR in patients with denosumab + ICI $>3$ months was 12.3, $\mathrm{P}=0.001(3.4-44.1)$.

\section{Discussion}

Denosumab is clinically indicated in NSCLC patients 

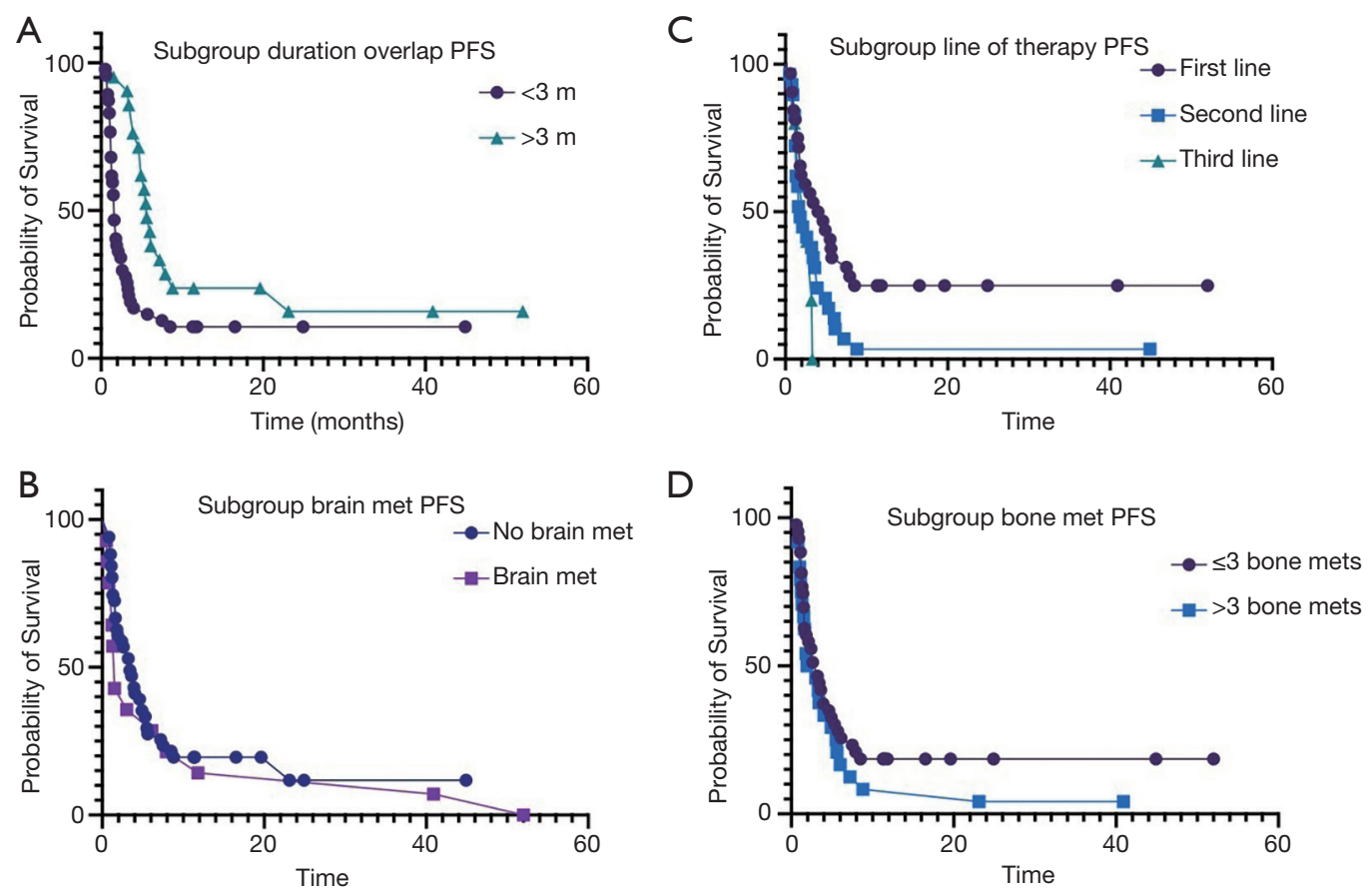

Figure 2 Kaplan-Meier estimate of PFS. (A) Kaplan-Meier estimate of PFS subgroups by duration of denosumab and ICI overlap (6.0 vs. 1.9 months $\mathrm{P}=0.0005)$. (B) Kaplan-Meier estimate of PFS subgroups by brain metastases (3.9 vs. 2.1 months, $\mathrm{P}=0.2$ ). (C) Kaplan-Meier estimate of PFS subgroups by line of therapy i.e., first line $v s$. second line (4.3 vs. 1.8 months, $\mathrm{P}=0.006)$. (D) Kaplan-Meier estimate of PFS subgroups by skeletal metastases ( 3.7 vs. 2 months, $\mathrm{P}=0.1$ ). ICI, immune checkpoint inhibitor.

Table 3 Best radiographic response

\begin{tabular}{lcc}
\hline Radiographic response & ORR & DCR \\
\hline $\mathrm{CR}=3(4.3 \%)$ & $18.8 \%$ & $40.6 \%$ \\
$\mathrm{PR}=10(14.5 \%)$ & & \\
$\mathrm{SD}=15(21.8 \%)$ & & \\
$\mathrm{PD}=41(59.4 \%)$ & & \\
\hline
\end{tabular}

$\mathrm{CR}$, complete response; PR, partial response; SD, stable disease; $\mathrm{PD}$, progressive disease.

with skeletal metastases. Here we report our experience combining denosumab with ICIs in metastatic NSCLC patients at a tertiary cancer center. Skeletal metastases are widely regarded as a negative prognosis marker in NSCLC. Skeletal metastases in malignant solid tumors involve the interplay of tumor cells and normal cells in bone microenvironment. In case of metastases, osteoclastic activity is increased, resulting in increased bone remodeling. This increased bone remodeling causes the bone destruction and skeletal complications. The upregulated bone turnover by osteoclastic bone resorption causes skeletal morbidity and early bone colonization of the metastatic tumor (11). Anti-RANKL is indicated to prevent skeletal related adverse events in lung cancer, and growing evidence suggests that RANK/RANKL signaling is involved in tumorigenesis (12). For example, RANK rewires energy hemostasis in murine and human lung cancer cell lines, and pharmacologic RANK inhibition decreases tumor growth in patientderived lung cancer xenografts (13).

ICIs have expanded the horizon of metastatic NSCLC treatment. ICIs were approved for the $2 \mathrm{nd}$ or 3 rd line treatment of metastatic NSCLC in patients without targetable driver mutations in 2015. Subsequently, ICIs have been approved in the 1 st line setting, either as a single agent in cancers with PD-L1 TPS of $>1 \%$ or in combination with chemotherapy independent of the PD-L1 status (14). Despite the durable antitumor response produced by ICIs in a growing list of various cancer types, the resistance to this therapy has motivated research on combinational therapies to enhance immune-surveillance and cancer control. Besides various combinations of ICIs 
with chemotherapy, targeted therapies and radiation, emphasis is placed on the identification of novel partners of ICIs and repurposing already-approved medications. Denosumab, an anti-RANKL monoclonal antibody (mAb), emerges as a potential repurposing target based on the roles of RANK/RANKL in tumorigenesis.

Preclinical and clinical studies have shown promising results combining RANKL inhibitor with ICIs in various cancer types including lung cancer. According to a posthoc analysis of phase III clinical trial in metastatic lung cancer patients, median overall survival was 8.9 months for patients receiving denosumab compared with 7.7 months for patients receiving zoledronic acid (10). Udagawa et al. reported that in chemotherapy naïve non-squamous nonsmall cell lung cancer patients with $80 \%$ having bone metastasis and unknown brain metastasis status, the median OS in the denosumab group, zoledronic acid group and no treatment group were 21.4 months, 12.7 months and 10.5 months, respectively (15). Liede and others reported that in NSCLC patients with $76 \%$ having Stage IV disease at diagnosis but unknown brain metastasis status, the ORR was $33.1 \%$ in patients treated concurrently with denosumab and anti-PD1 mAb (16). In our study, we reported a median OS of 6.3 months with ORR of $18.8 \%$. However, due to vast variability in terms of baseline patient characteristics including brain and bone metastasis as well as prior treatments, cross-sectional comparison is challenging among these studies.

There are multiple clinical trials to investigate the anticancer property of denosumab. In the ongoing phase Ib/ II POPCORN trail, pre-operative nivolumab with and without denosumab is studied in resectable NSCLC (17). In the phase II SPLENDOUR trial in stage IV NSCLC, median OS was 8.7 months in the chemotherapy alone arm versus 8.2 months in the chemotherapy-denosumab arm (18). However, there is no clinical trial ongoing that test the combination of ICIs with denosumab in NSCLC, although similar trials are underway in metastatic melanoma and renal cell carcinoma (12).

Among our patients, $37.7 \%$ of the patients have brain metastases. Brain metastases have historically been listed as exclusion criteria in previous pivotal clinical trials involving ICIs in advanced NSCLC. The historically documented 2 -year survival for patients with brain metastases from NSCLC is $14.3 \%$ (19). Despite the presence of aggressive features such as brain metastases and multiple bone metastases, our study population achieved an overall survival of 6.3 months. We did not observe statistically worse OS and PFS in patients with brain metastases comparing to those without brain metastases, nor did we note worse outcome in patients with $>3$ skeletal metastases comparing to those with $<3$ metastatic sites. It could be due to our limited sample size. Alternatively, the result suggests a potential benefit of the longer duration of denosumab in the metastatic settings.

Published response rates in advanced NSCLC (not selected by PD-L1 expression) to anti-PD1 ICI monotherapy range from $18-20 \%$ for second line treatment to $23-24.8 \%$ for first-line treatment (16). In published trials of chemotherapy and ICI combinations, PFS ranges from 5.1-17.2 months for first line therapy and $2.3-3.9$ months in second line therapy (20). In this cohort, $43.5 \%$ of patients received second line treatment and our ORR of $18.8 \%$ and PFS of 2.8 months fall within the range of second line treatment. There is also no difference in OS and PFS between elderly ( $>75$ years old) and younger population, indicating that denosumab is safe to be combined with ICIs in elderly population who are more prone for skeletal adverse events. Our study spans from 2015 to 2020 with notable guideline updates such as the approval of pembrolizumab plus pemetrexed and platinum-based chemotherapy. This study demonstrated, that first line ICI therapy is superior to second and third line therapies. We also observed that the duration of ICIs and denosumab overlap had a significant impact on OS and PFS. Of note, among the 13 patients who achieved CR and PR, six-month survival rate was $100 \%$ and one-year survival rate was $69.2 \%$. Thus, the correlation can be explained by patients who responded to ICI/denosumab combination therapy were able to achieve durable responses and continue denosumab treatment for a prolonged time. Future studies are needed to further elucidate the relationship between duration of combination therapy and treatment responses.

Recognizing the limitations of this study as a single center retrospective observational study with small sample size, it provides real world experience combining ICIs with denosumab in Stage IV NSCLC patients with significant brain and skeletal metastatic burden, as well as considerable proportion receiving ICI as second line of therapy.

\section{Conclusions}

In this study, we reported that OS and PFS were comparable to the published studies, however overall our patients had more aggressive disease. Duration of ICIs and denosumab overlap has a significant positive impact on OS and PFS. 
Denosumab is a safe option that is overall well tolerated. However, future studies are needed to further characterize the therapeutic potential of denosumab beyond known prevention of skeletal related complications when used in combining with ICIs in metastatic NSCLC.

\section{Acknowledgments}

Funding: None.

\section{Footnote}

Reporting Checklist: The authors have completed the STROBE reporting checklist. Available at https://dx.doi. org/10.21037/jtd-21-150

Data Sharing Statement: Available at https://dx.doi. org/10.21037/jtd-21-150

Conflicts of Interest: All authors have completed the ICMJE uniform disclosure form (available at https://dx.doi. org/10.21037/jtd-21-150). The authors have no conflicts of interest to declare.

Ethical Statement: The authors are accountable for all aspects of the work in ensuring that questions related to the accuracy or integrity of any part of the work are appropriately investigated and resolved. This study was approved by institutional review board (IRB) of DartmouthHitchcock Medical center/Dartmouth College. Due to retrospective nature of the study, request for informed consent waiver was approved by IRB (ID of ethics approval: STUDY00030057).

Open Access Statement: This is an Open Access article distributed in accordance with the Creative Commons Attribution-NonCommercial-NoDerivs 4.0 International License (CC BY-NC-ND 4.0), which permits the noncommercial replication and distribution of the article with the strict proviso that no changes or edits are made and the original work is properly cited (including links to both the formal publication through the relevant DOI and the license). See: https://creativecommons.org/licenses/by-nc-nd/4.0/.

\section{References}

1. Siegel RL, Miller KD, Jemal A. Cancer statistics, 2020. CA Cancer J Clin 2020;70:7-30.
2. National Cancer Institute. SEER Cancer Statistics Review, 1975-2011. Available online: http://seer.cancer.gov/ csr/1975_2011/

3. Brahmer J, Reckamp KL, Baas P, et al. Nivolumab versus Docetaxel in Advanced Squamous-Cell Non-Small-Cell Lung Cancer. N Engl J Med 2015;373:123-35.

4. Gandhi L, Rodríguez-Abreu D, Gadgeel S, et al. Pembrolizumab plus Chemotherapy in Metastatic NonSmall-Cell Lung Cancer. N Engl J Med 2018;378:2078-92.

5. Lacey DL, Boyle WJ, Simonet WS, et al. Bench to bedside: elucidation of the OPG-RANK-RANKL pathway and the development of denosumab. Nat Rev Drug Discov 2012;11:401-19.

6. Henry DH, Costa L, Goldwasser F, et al. Randomized, double-blind study of denosumab versus zoledronic acid in the treatment of bone metastases in patients with advanced cancer (excluding breast and prostate cancer) or multiple myeloma. J Clin Oncol 2011;29:1125-32.

7. Gül G, Sendur MA, Aksoy S, et al. A comprehensive review of denosumab for bone metastasis in patients with solid tumors. Curr Med Res Opin 2016;32:133-45.

8. Coleman RE. Management of bone metastases. Oncologist 2000;5:463-70.

9. De Castro J, García R, Garrido P, et al. Therapeutic Potential of Denosumab in Patients With Lung Cancer: Beyond Prevention of Skeletal Complications. Clin Lung Cancer 2015;16:431-46.

10. Scagliotti GV, Hirsh V, Siena S, et al. Overall survival improvement in patients with lung cancer and bone metastases treated with denosumab versus zoledronic acid: subgroup analysis from a randomized phase 3 study. J Thorac Oncol 2012;7:1823-9.

11. Weilbaecher KN, Guise TA, McCauley LK. Cancer to bone: a fatal attraction. Nat Rev Cancer 2011;11:411-25.

12. Ahern E, Smyth MJ, Dougall WC, et al. Roles of the RANKL-RANK axis in antitumour immunity - implications for therapy. Nat Rev Clin Oncol 2018;15:676-93.

13. Rao S, Sigl V, Wimmer RA, et al. RANK rewires energy homeostasis in lung cancer cells and drives primary lung cancer. Genes Dev 2017;31:2099-112.

14. Sui H, Ma N, Wang Y, et al. Anti-PD-1/PD-L1 Therapy for Non-Small-Cell Lung Cancer: Toward Personalized Medicine and Combination Strategies. J Immunol Res 2018;2018:6984948.

15. Udagawa $\mathrm{H}$, Niho $\mathrm{S}$, Kirita K, et al. Impact of denosumab use on the survival of untreated non-squamous non-small cell lung cancer patients with bone metastases. J Cancer 
Res Clin Oncol 2017;143:1075-82.

16. Liede A, Hernandez RK, Wade SW, et al. An observational study of concomitant immunotherapies and denosumab in patients with advanced melanoma or lung cancer. Oncoimmunology 2018;7:e1480301.

17. Ahern E, Cubitt A, Ballard E, et al. Pharmacodynamics of Pre-Operative PD1 checkpoint blockade and receptor activator of NFkB ligand (RANKL) inhibition in nonsmall cell lung cancer (NSCLC): study protocol for a multicentre, open-label, phase $1 \mathrm{~B} / 2$, translational trial (POPCORN). Trials 2019;20:753.

18. Peters S, Danson S, Hasan B, et al. A Randomized Open-Label Phase III Trial Evaluating the Addition of

Cite this article as: Cao Y, Afzal MZ, Shirai K. Does denosumab offer survival benefits? -Our experience with denosumab in metastatic non-small cell lung cancer patients treated with immune-checkpoint inhibitors. J Thorac Dis 2021;13(8):4668-4677. doi: 10.21037/jtd-21-150
Denosumab to Standard First-Line Treatment in Advanced NSCLC: The European Thoracic Oncology Platform (ETOP) and European Organisation for Research and Treatment of Cancer (EORTC) SPLENDOUR Trial. J Thorac Oncol 2020;15:1647-56.

19. Ali A, Goffin JR, Arnold A, et al. Survival of patients with non-small-cell lung cancer after a diagnosis of brain metastases. Curr Oncol 2013;20:e300-6.

20. Wagner G, Stollenwerk HK, Klerings I, et al. Efficacy and safety of immune checkpoint inhibitors in patients with advanced non-small cell lung cancer (NSCLC): a systematic literature review. Oncoimmunology 2020;9:1774314. 